\title{
Substructure analysis by means of neutron diffraction
}

\author{
P. KLIMANEK, T. KSCHIDOCK, P. LUKAS*, P. MIKULA* , A. MÜCKLICH** and M. VRANA*
}

Freiberg University of Technology, Department of Materials Science, 9596 Freiberg/Sa., Germany

* Nuclear Physics Institute, CR-25 068 Rez n. Prague, Czech Republic

** Research Center Rossendorf, Institute of Ion Physics and Materials, 11314 Dresden, Germany

\begin{abstract}
Shape analysis of neutron diffraction peaks was used for substructure characterization in plastically deformed, polycrystalline ferritic steel. The high resolution of the scattering device used allows a quantitative estimation of substructure parameters as e.g. dislocation densities. The resultes are in good agreement with those obtained in $x$-ray investigations of the material and indicate that high-resolution neutron diffraction is a useful completion of integrated substructure analysis.
\end{abstract}

\section{INTRODUCTION}

Integrated substructure analysis in polycrystalline materials (i.e. analysis of lattice disorder of the 2nd kind due to structure defects as dislocations, stacking faults, subboundaries etc.) can be performed by evaluation of the line broadening of $x$ - ray diffraction peaks. In this connection two difficulties have often to be taken into account: - the limited number of grains which contribute to the observable line shape and broadening and, consequently, to the orientation averaging over the lattice disorder of the individual crystallites, and

- the strong decrease of the X-ray scattering intensities at higher diffraction angles, which significantly complicates the analysis of higher diffraction orders.

Because elastic scattering of neutrons by polycrystals is, in general, the result of averaging over a large number of crystallites, and since the atomic scattering power of the sample material is, as a rule, independent on the scattering angle, shape analysis of physically broadened neutron diffraction peaks should be an attractive completion of the existing procedures of integrated substructure investigation. In this connection further advantages of neutron diffraction may be mentioned: the large scattering volume, which is representative for a macroscopic 
specimen, applicability for coarse-grained samples $\left(D_{\mathrm{x}}>100 \mu \mathrm{m}\right)$, absence of surface effects, high peak-background intensity ratio, and the possibility of investigation of real components.

of course, in order to realize sufficiently precise line shape measurements high-resolution neutron diffraction has to be available.

That diffraction-line broadening due to lattice disorder of the 2nd kind can be investigated by neutron diffraction was shown, for instance, by measurements of rocking curves of individual crystallites in a plastically deformed nickel alloy [1]. First data concerning the quantitative evaluation of polycrystal reflections were reported in [2]. In this paper resultes of a more systematic investigation and a comparision between $\mathrm{X}$-ray and neutron diffraction analysis are presented.

\section{METHODOLOGICAL BACKGROUND}

The observable line shape of a neutron diffraction peak $F(x)$ due to a polycrystalline sample material is, as in conventional X-ray powder diffractometry, always the convolution of an instrumental line profile $g(u)$ due to the nonideal conditions of the scattering experiment and a physical line profile $f(x)$ due to the lattice disorder of the 2 nd kind. Accordingly, as in $x$-ray diffraction the analysis of broadened neutron reflections encloses

* the separation of the distributions $g(y)$ and $f(x)$,

* identification of the types of lattice disorder of the 2nd kind being present and separation of the corresponding contributions to the physical line broadening, and

* quantitative estimation of substructure parameters (e.g. grain or subgrain sizes, dislocation densities, stacking-fault probabilities etc.).

The first problem can be approximately solved on the base of the Fourier transformation (stokes correction [3]). For this purpose the line shape $g(u)$ must be determined by means of a standard sample of the interesting or a sufficiently similar material, the lattice disorder of whose crystallites can be neglected.

In the evaluation of the physical line profile $f(x)$, which should generally be based on the Fourier coefficients $A(L)$ ( $L=$ nd $_{h k l}$ length perpendicular to the reflecting lattice planes (hkl)), the following components of line broadening are important :

* particle-size broadening due to interfaces

* strain broadening due to internal stresses of the 2 nd kind which are constant within crystallites or subgrains, and

* line broadening due to dislocations.

Using the results of theoretical work concerning these phenomena it can be shown $[4,5]$ that the physical line broadening is characterized by the functions

$\Phi(L)=-\ln A(L) / L$

and

$$
\begin{aligned}
\Psi(L)=-\ln A(L) / L^{2} & =1 / T L+\left(K<\epsilon^{2}>+B \ln L_{0}\right)-B \operatorname{lnL} \\
& =1 / T L+K<e^{2}(0)>-B \operatorname{lnL}
\end{aligned}
$$


with $T=T(h k l)$ - effective particle size, $B=B(h k l)$ - factor proportional to the mean total dislocation density $N, L_{0}$ - length proportional to the outer cut-off radius $R$ of the strain field of a dislocation, and $\left\langle\epsilon^{2}\right\rangle=\left\langle\epsilon^{2}(\mathrm{hkl})\right\rangle-$ mean square strain due to internal stresses of the 2nd kind.

The graphs of $\phi(L)$ and $\Psi(L)$ were called a Warren-Averbach plot and a Krivoglaz-Wilkens plot, respectively. From $\phi(L)$ the reciprocal particle size $1 / T$ and an effective mean square strain $\left\langle e^{2}\right.$ (hkl) $\rangle$ can be determined and from $\Psi(L)$, which should have a linear branch at higher $L$, the quantity $\mathrm{B}(\mathrm{hkl})$ and the dislocation density $\mathrm{N}_{\mathrm{d}}$, respectively, are obtained. If the strain component $\left\langle\epsilon^{2}\right\rangle$ is sufficiently small, the parameter $L_{0}$ can also be estimated.

In the present paper only the determination of dislocation densities $\mathrm{N}_{\mathrm{a}}$ from the parameter $\mathrm{B}$ is briefly considered. This can be done by means of the relationship (b- amount of the Burgers vector, $\chi_{0}(\mathrm{hkl})$ factor depending on the orientations between the slip systems $\{h k l\}<u v w>$ of the dislocations and the operating diffraction vector $h$, $h=2 \sin \theta / \lambda=1 / d(h k l)$

$$
B(h k l)=B_{0}(h k l) N_{d}=(\pi / 2) b^{2} h^{2} \chi_{0}(h k l) N_{d}
$$

which is valid for dislocation arrangements with weak defect correlation in elastically isotropic cubic crystals $[6,7,8]$.

Numerical values of $\chi_{0}(\mathrm{hkl})$ for screw and edge dislocation with Burgers vectors $b=1 / 2\langle 111\rangle$ in $b . c . c$. polycrystals with randomly oriented grains are given in [9]. Thus, assuming that essentially screw dislocations contribute to the line broadening, the following values $B_{0}$ (hkl) are obtained: $B_{0}(110)=0.262\left[\mathrm{~nm}^{-2}\right], B_{0}(200)=0.785\left[\mathrm{~nm}^{-2}\right]$, and $B_{0}^{0}(211)$ $=0.785\left[\mathrm{~nm}^{-2}\right]$.

\section{EXPERTMENTAL PROCEDURES}

Sample material: The sample material of the work presented here was a well-annealed $\left(850^{\circ} \mathrm{C} / 30 \mathrm{~min}\right)$ high-alloy ferritic steel X8CrTil7 with a mean grain size of $20 \mu \mathrm{m}$, which was plastically deformed by compression at room temperature $(\epsilon=5-80 \%)$. The small grain size was chosen in order to improve the comparibility of the results of X-Ray and neutron diffraction analysis. Moreover, the samples for both diffraction techniques were prepared in such a manner that the influence of the deformation texture was reduced.

Neutron-diffraction: The neutron diffraction experiments were performed with the high-resolution triple axis spectrometer TKSN 400 of the Nuclear Physics Institute at Rez $n$. Prague, which uses bent Si single crystals. A detailed description of the instrument is given in $[10,11]$; the conditions of the work described here are summarized in Table 1 . $x$-ray analysis: $X$-ray diffraction profiles were measured by means of a powder diffractometer HZG4 of the Freiberger Präzisionsmechanik $\mathrm{GmbH}$ with $\mathrm{Co}-\mathrm{K}_{a}$ radiation. In order to reduce the instrumental line broadening and the influence of background scattering, soller slits and secondary-beam monochromatization were used. As in neutron diffraction the standard of the $\mathrm{X}$-ray profile analysis was well-annealed $\alpha$-iron. Data evaluation: Because particularly the interpretation of a Krivoglaz-Wilkens plot requires sufficient accuracy of the high-order Fourier coefficients, these were not calculated directly from the experimental data but after careful fitting of the line shapes $F(x)$ and $g(u)$ together with the background scattering. 
Table 1

\begin{tabular}{|c|c|c|c|c|c|c|c|}
\hline $\begin{array}{l}\text { Monochromator-analyzer } \\
\text { combination : }\end{array}$ & Ferrite & $\begin{array}{l}(110) \\
(211)\end{array}$ & M: & $\begin{array}{l}\mathrm{Si} \\
\mathrm{Si}\end{array}$ & $\begin{array}{l}(111) \\
(220)\end{array}$ & A: & $\begin{array}{l}\mathrm{Si} \\
\mathrm{Si}\end{array}$ \\
\hline $\begin{array}{l}\text { Neutron } \\
\text { wavelength : } \\
\text { Standard material : } \\
\text { Scattering volume : } \\
\text { Registration mode : }\end{array}$ & $\begin{array}{r}\text { Ferri } \\
\alpha- \\
\text { step }\end{array}$ & $\begin{array}{l}\text { te }\left(\begin{array}{l}(110) \\
(211)\end{array}\right. \\
\text { Iron } \\
\text { scanning; }\end{array}$ & $\begin{array}{r}V \\
\delta \theta\end{array}$ & $\begin{array}{l}= \\
=\end{array}$ & $\begin{array}{r}0.16 \\
0.13 \\
\\
160 \\
0.018\end{array}$ & $\begin{array}{l}\mathrm{nm} \\
\mathrm{nm} \\
\mathrm{mm}^{3} \\
0\end{array}$ & \\
\hline
\end{tabular}

Counts $\left({ }^{*} 1000\right)$

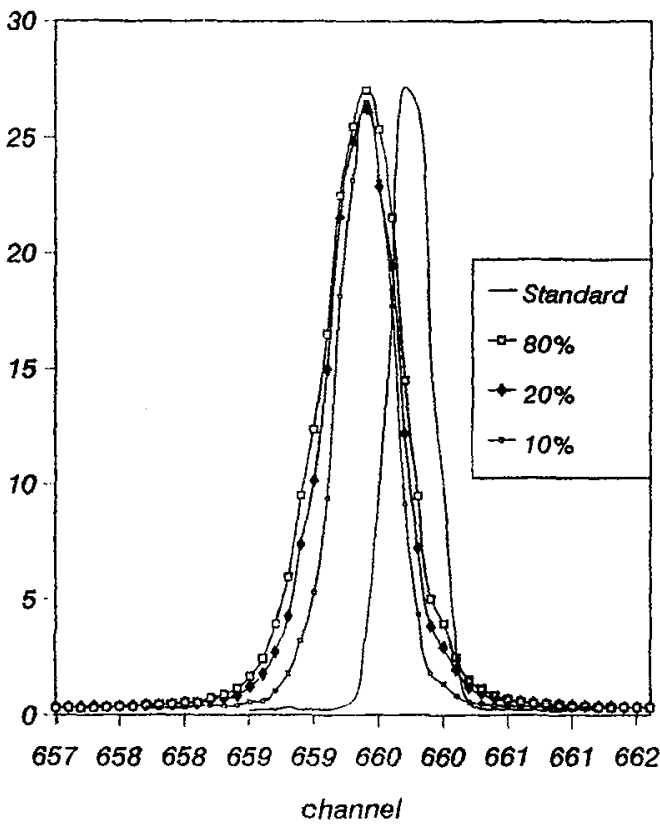

Fig.1 a- (110)neutron peak,

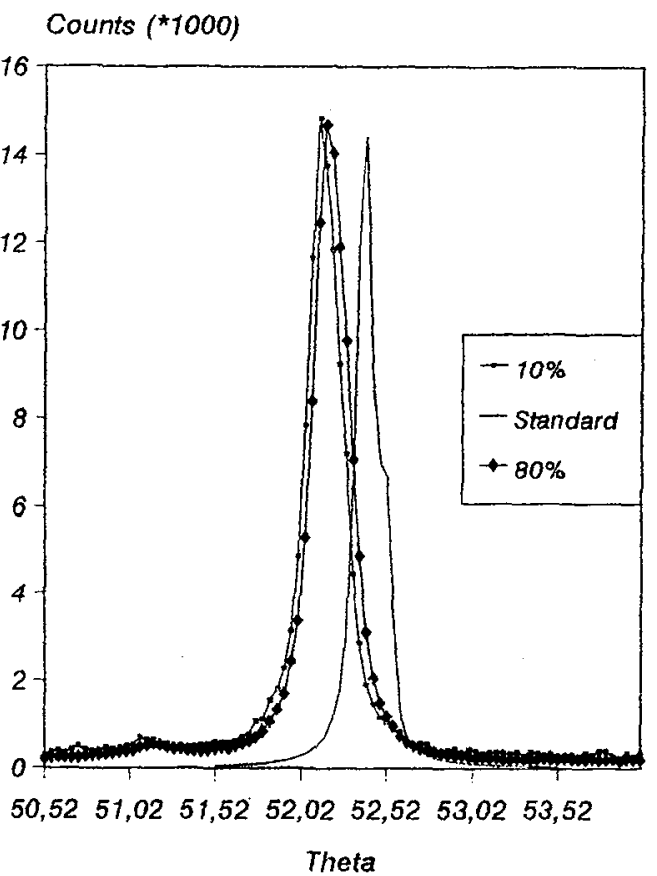

b- (110)X-ray peak

\section{RESULTS AND DISCUSSION}

In Fig. 1 the line shapes of the (110) reflections as obtained by X-ray and neutron scattering are presented. In both cases the increasing line broadening with increasing deformation degree is clearly visible and comparable but, of course, relatively low because of the low scattering angle. Moreover, Fig. la shows that the instrumental line broadening of the neutron reflections is remarkable small.

The evaluation of the line broadening may be illustrated by fig. 2 which shows the Krivoglaz-Wilkens plot of a neutron reflection. The dislocation parameters $B$ and dislocation densities $N_{\alpha}$ determined from the Krivoglaz-Wilkens plots of the neutron reflections (211) are summarized in Table 2 together with those obtained by $x$-ray analysis. 
Table 2

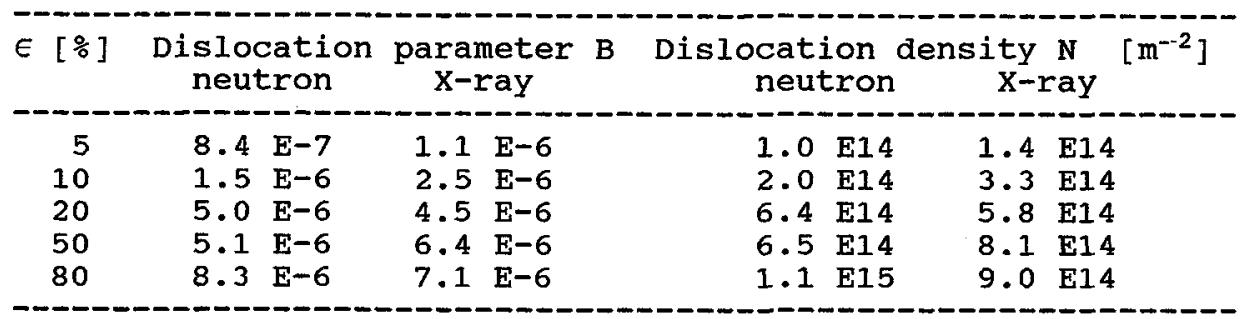

The agreement between the results of neutron and $x$-ray profile analysis is very good; in order to explain the differences the assumption of an experimental uncertainty $\delta \mathrm{B} / \mathrm{B} \leq 20 \%$ is sufficient.

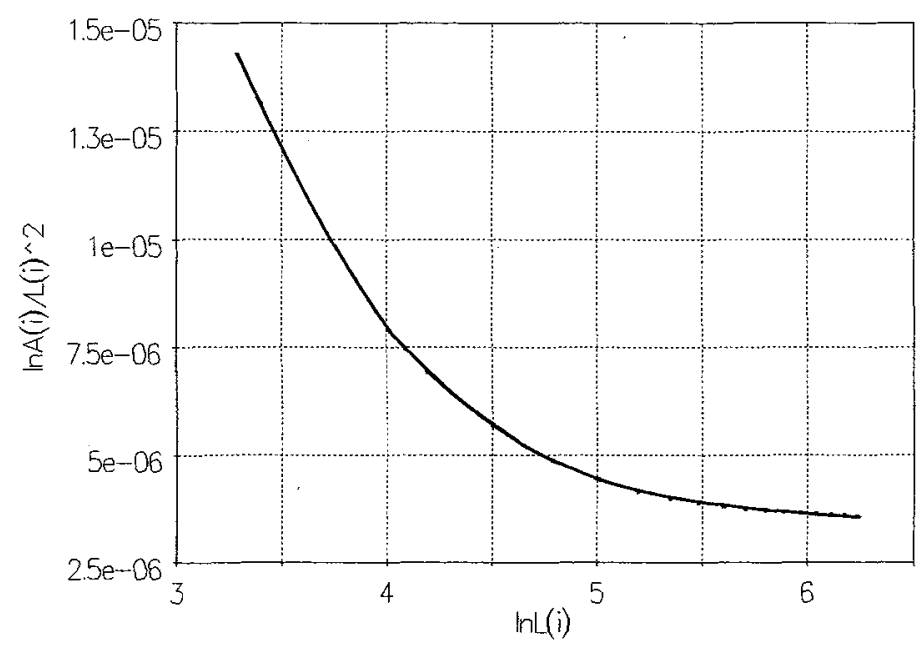

Fig. 2: Krivoglaz-Wilkens plot ( $\epsilon-20 \%,(110)$-reflection)

In Fig. 3 the dislocation densities $\mathrm{N}_{d}$ estimated from the line broadening of the reflections (110) and (211) are compared. It is visible that

* the agreement between neutron and $X$-ray profile analysis is less good in the case of the (110) reflections than in the case of the (211) diffraction peaks, and that

* the dependence of $\mathrm{N}_{\mathrm{d}}$ on the deformation degree $\epsilon$ seems to be different for both reflections.

The first phenomeneon can be caused by several effects: uncertainties of the Krivoglaz-Wilkens plots (in $\mathrm{x}$-ray and neutron diffraction) and structural inhomogeity (cp. $[4,5]$ ) and/or insufficient surface preparation of the samples in $\mathrm{x}$-ray analysis. Its correct explanation requires further experimental work. The second effect, $N_{d}(211)>N_{d}(110)$, was also observed in X-ray analysis of tensile specimens [9] and qualitatively related to the texture of the sample material, but a detailed interpretation is outstanding. 


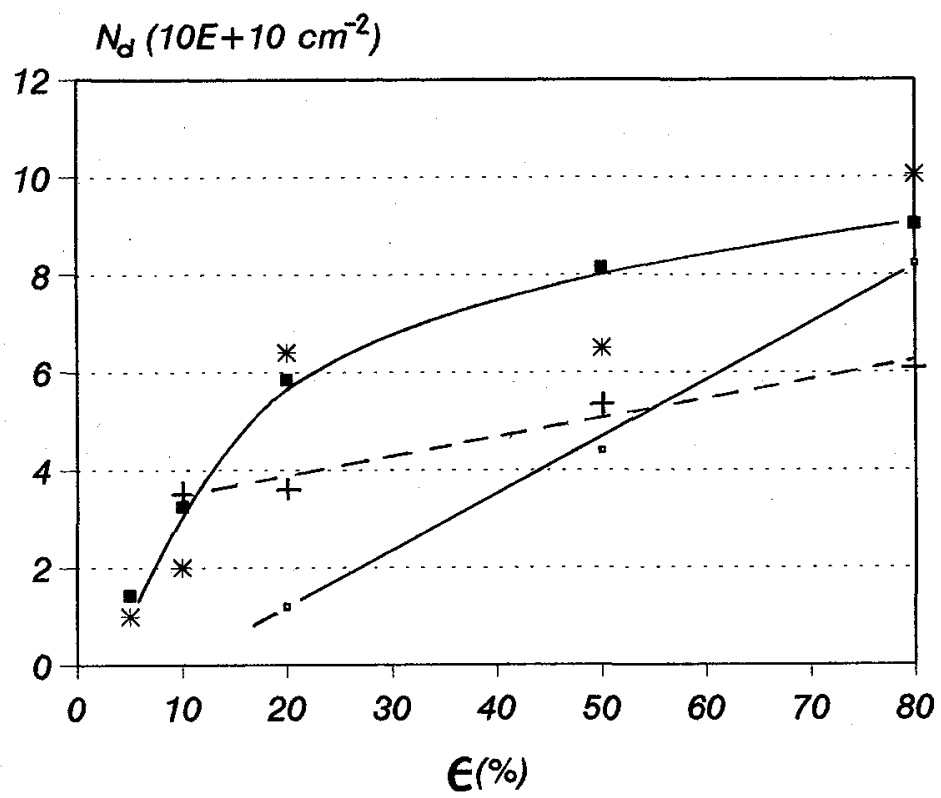

- (110)-neutron

$+(110)-X$-ray

* (211)-neutron

- (211)-X-ray

Fig.3: Dislocation density vs deformation degree

The present work was supported by the Federal Ministery for Research and Technology (BMFT) of the FRG.

\section{References}

[ 1] CROSTACK H. -A., REIMERS W., SELVADURAI U., ECKOLD G.: Nondestructive Characterization of Materials (Ed.P. Möller et al.) Springer-Verlag, Berlin (1989), 323-330

[ 2] KLIMANEK P., KSCHIDOCK T., MÜCKLICH A., MIKULA P., VRANA M.: EPDIC 2 - European Powder Diffraction (Editors:R. DELHEZ, E.J. MITTEMETJER) . Mat. Sci. Forum (1993), in print

[ 3] STOKES A.R. : Proc.Phys.Soc. (London) 61 (1948) 3382

[ 4] KLIMANEK P. : X-Ray and Neutron Structure Analysis in Materials Research (Ed. J. HASEK), Plenum Press, New York (1989), 125-137

[ 5] KLIMANEK P.: EPDIC I - European Powder Diffraction (Editors: R. DELHEZ, E.J. MITTEMEIJER). Mat. Sci. Forum 79-82 (1991) Pt. I, $73-84$

[ 6] WILKENS M. : phys.stat.sol.(a) 2 (1970) 359

[ 7] WILKENS M. : Kristall \& Technik 11 (1976) 1159

[ 8] KRIVOGLAZ M.A., MARTYNENKO O.V., RYABOSHAPKA K.P.: Fizika Metallov i Metalloved. 55(1983) 5

[ 9] KLIMANEK P.: this issue

[10] KULDA J., MIKULA P., LUKAS P., KOCSIS M.: Proc. Int. Conf. on Neutron Scattering, oxford (1991), Physica B,

[11] ALEFELD B., KLIMANEK P., KULDA J., et.al.: this issue 